\title{
Polarization interferometer using a Liquid Crystalline Polymer waveplate for wavelength measurement
}

\author{
Paweł Wierzba, Adam Mazikowski \\ Department of Metrology and Optoelectronics, Faculty of Electronics, Telecommunications and Informatics, \\ Gdańsk University of Technology, Narutowicza 11/12, 80-233 Gdańsk,
}

Received November 15, 2015; accepted November 23, 2015; published December 31, 2015

\begin{abstract}
A two-beam polarization interferometer for measuring wavelength changes of a tunable semiconductor laser was designed, built and tested. This interferometer uses a $\lambda / 8$ waveplate made from Liquid Crystalline Polymer in order to generate two output signals in quadrature. The $\lambda / 8$ waveplate can be manufactured either on a beamsplitter face or on the mirror surface, reducing the cost and complexity of the optomechnical assembly. Experiment results show that a resolution of $2 \mathrm{pm}$ was attained for the wavelength range 628 $635 \mathrm{~nm}$. The range of operation of such an interferometer can be extended if the wavelength-dependent Heydemann correction is used.
\end{abstract}

Liquid Crystalline Polymers (LCPs) have been steadily expanding their area of application in photonics. In particular, several innovative optical components made from LCPs were developed, such as spiral phase plates, patterned polarizers and vortex retarders. Having unique properties, these components have been employed in sensing and metrology applications, such as astronomy, polarimetry, and microscopy (cf. e.g. [1], [2] and [3], respectively), enabling the development of new scientific instruments and measurement techniques.

Components made from LCPs have also a substantial application potential in polarization interferometry. The case in point is the $\lambda / 8$ waveplate, which can be employed in polarization interferometers measuring displacement or wavelength, especially for operation in the UV-VIS spectral region, where a zero-order crystalline waveplate is difficult and expensive to produce.

A two-beam polarization interferometer for the measurement of a wavelength emitted by an external cavity laser was designed, following a request from members of our research team. A measurement range of $0.1 \mathrm{~nm}$ was needed, along with the ability to track the changes in the wavelength in the $628 \mathrm{~nm}-635 \mathrm{~nm}$ range. The expected resolution was about $0.002 \mathrm{~nm}$.

The designed interferometer, whose layout is shown in Fig. 1, is a modification of the interferometers described in [4]. A $\lambda / 8$ waveplate made from LCP is used to obtain a $\pi / 4$ phase shift between the two orthogonally polarized components of the beam propagating in one arm of the interferometer.

\footnotetext{
*E-mail: pwierzba@eti.pg.gda.pl
}

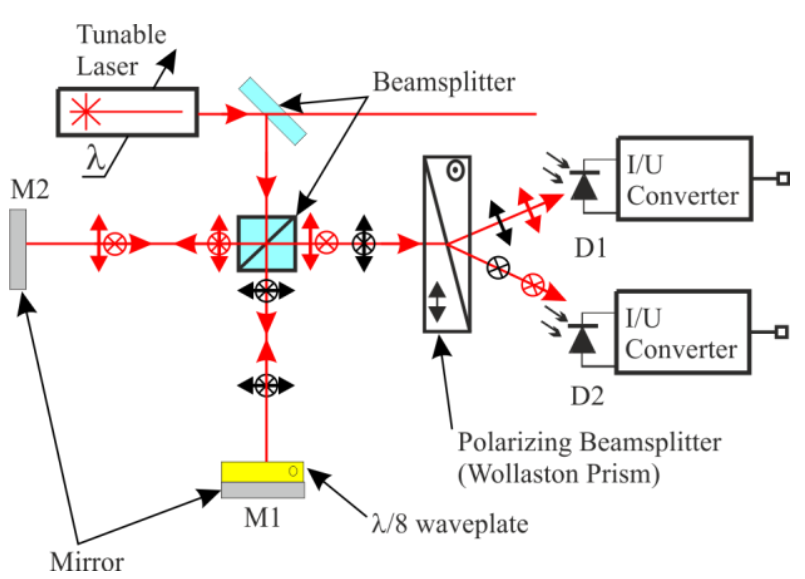

Fig. 1. Polarization interferometer for measurement of wavelength changes of a tunable semiconductor laser. D1, D2 - photodiodes.

When such an interferometer is illuminated with a monochromatic beam of intensity $I_{0}$, the intensity of the light incident on detectors $\mathrm{D}_{1}$ and $\mathrm{D}_{2}$ can be expressed as:

$$
\begin{aligned}
& I_{1}=\frac{I_{0}}{4}[1+\cos \varphi], \\
& I_{2}=\frac{I_{0}}{4}[1+\sin \varphi],
\end{aligned}
$$

where $\varphi$ - phase difference of interfering beams, given by:

$$
\varphi=\frac{2 \pi}{\lambda} \Delta l,
$$

where $\lambda-$ the wavelength of the beam, $\Delta l$ - the optical path length difference between the two arms of the interferometer. By changing $\Delta l$, the measurement range of the wavelength $\Delta \lambda$ can be adjusted.

The value of optical path length difference $\Delta l$ corresponding to required $\Delta \lambda$ can be calculated using Eq. (2) by setting the change of phase $\varphi$ corresponding to $\Delta \lambda$ equal to $2 \pi$, i.e:

$$
\Delta \varphi=2 \pi=\frac{2 \pi}{\lambda_{C}} \Delta l-\frac{2 \pi}{\lambda_{C}+\Delta \lambda} \Delta l,
$$

where $\Delta \varphi-$ the change of phase, $\lambda_{C}-$ the central wavelength of an operating range of the interferometer, $\Delta l$ - the optical path length difference, $\Delta \lambda$ - the measurement 
range of the wavelength. For $\lambda_{C}=633.5 \mathrm{~nm}$ and $\Delta \lambda=0.1 \mathrm{~nm}$, $\Delta l$ equals $3.99 \mathrm{~mm}$.

The LCP waveplates were manufactured using technology similar to that described in detail in Ref. [5]. Two essential modifications were introduced in the process flow shown schematically in Fig. 3. First, the heating step between spin-coating and UV curing was removed, as it resulted in several point- and bubble-like defects and discontinuities of the LCP surface. Instead, a gentle blow of warm air $\left(25-35^{\circ} \mathrm{C}\right)$ was used before and during curing to promote the solvent evaporation. Second, high power UV LEDs whose central wavelength was $385 \mathrm{~nm}$ were used as the UV curing lamps.

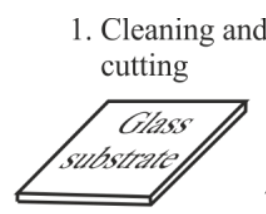

\section{Alignment layer 3. Rubbing} deposition
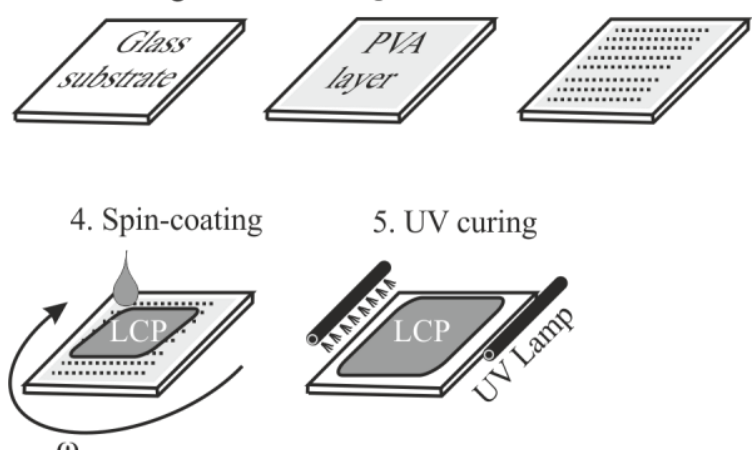

Fig. 2. Manufacturing technology of LCP waveplates.

A series of several LCP waveplates were made on microscopy glass substrates cut to $25 \mathrm{~mm}$ by $25 \mathrm{~mm}$ size. The quality of LCP layers was good, virtually without defects in the central part of the waveplate. Similarily, the uniformity of retardation in the central part of the waveplate was within $5 \%$ of the mean value. Overall, the optical quality of the waveplates is good, as can be seen in Fig. 3.

It should be noted that in commercially produced equipment the waveplate can be manufactured either on the beamsplitter face or on the mirror surface, thereby reducing the complexity and cost of the optomechnical assembly as well as facilitating the alignment of the optical setup.

The only exceptions are the left- and right-hand corner areas, where the LCP layer becomes thicker and its alignment is not as good as in the central part of the waveplate.

The process parameters, such as rotation speed and the amount of solvent were selected, based on our previous experience, to yield waveplates with $\pi / 4$ retardation at $630 \mathrm{~nm}$. The retardation of the waveplates was measured using a He-Ne laser and a Thorlabs PAX5710VIS polarimeter. A waveplate whose retardation was within
$2 \%$ of the nominal $\pi / 4$ value was selected for use in the interferometer.

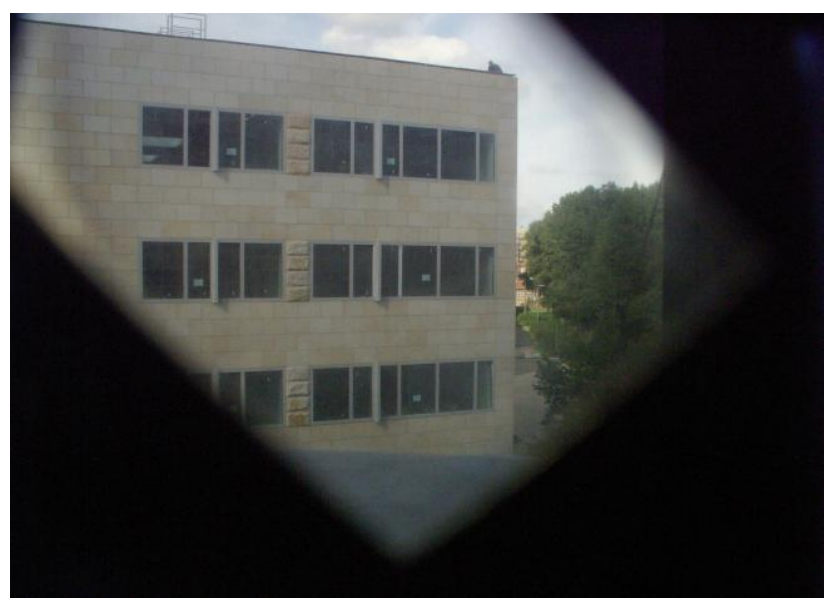

Fig. 3. Building seen through an LCP waveplate placed between crossed polarizers.

The interferometer was built on an optical plate using off-the-shelf optical and optomechanical components. A cube beamsplitter BS was used to reduce the influence of dispersion by ensuring that the beams in both arms propagate through the same distance in glass. For alignment, a New Focus 6304 tunable laser was connected, via a collimator, to the input of the interferometer, as shown in Fig. 4.

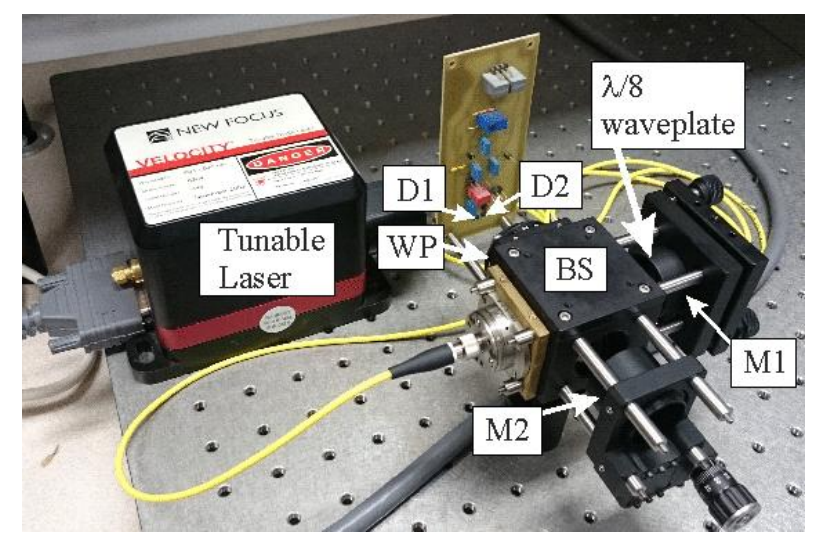

Fig. 4. Polarization interferometer during the alignment procedure. M1, M2 - mirrors, BS - beamsplitter, D1, D2 - photodiodes, WP - Wollaston prism.

The alignment comprised of three steps. First, the mirrors were aligned so that an interference signal was obtained at detectors D1 and D2. In the second step, the optical paths in both arms were made equal. Initial setting was performed using a dial caliper, followed by precision adjustment done by the micrometer actuator in the mount of mirror M2. During the precision adjustment the laser was tuned in a range of $2 \mathrm{~nm}$ to detect the position of 
mirror M2 in which the output signal remained constant. In the third step, the mount with mirror M2 was pushed forward by a differential micrometer head, by $1.995 \mathrm{~mm}$, in order to introduce the optical path length difference $\Delta l=3.99 \mathrm{~mm}$.

Next, the laser connected to the interferometer was tuned by $1 \mathrm{~nm}$ and the signals Ux and Uy corresponding to intensity $I_{1}$ and $I_{2}$, respectively, were recorded. The Heydemann correction [6] was applied, and signals Ux and Uy after correction, corresponding to a wavelength change of $0.1 \mathrm{~nm}$, are shown in Fig. 5.

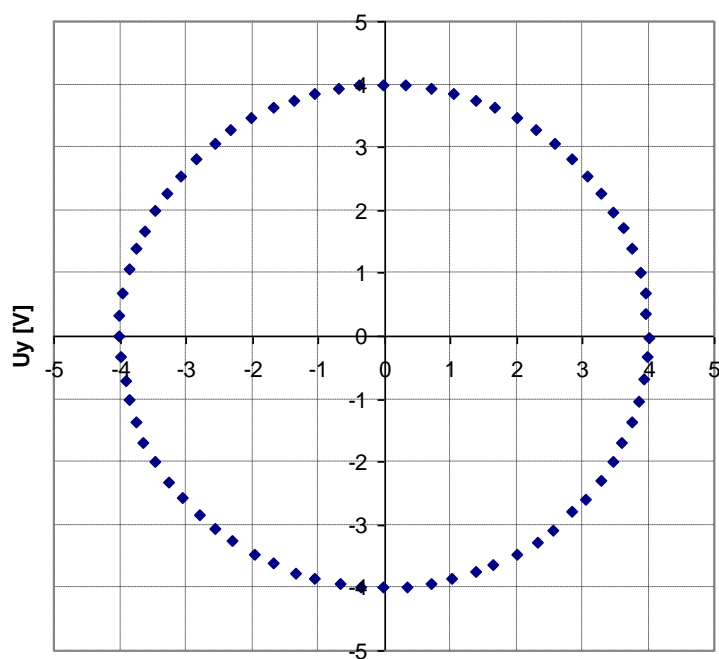

$\mathrm{Ux}[\mathrm{V}]$

Fig. 5. Output signals Ux and Uy from the interferometer after removal of the constant components and application of the Heydemann correction .

Based on the analysis of the acquired data, and taking into account the noise of the current-to-voltage converters, it can be concluded that the required resolution of $0.002 \mathrm{~nm}$ was attained. Determination of accuracy is much more difficult, as it would require comparison with a wavelength meter, not available to the Authors of this Letter.

A disadvantage of the presented interferometer is the dependence of the phase change introduced by the $\lambda / 8$ waveplate on the wavelength. In this particular case, the maximum change in the wavelength is $3.5 \mathrm{~nm}$ from its central value. In the absence of LCP birefringence dispersion, this would correspond to the phase change $\delta \varphi$ of $8.7 \mathrm{mrad}$, i.e. $0.50^{\circ}$. In the target application the error introduced by this $\delta \varphi$ is acceptable, and any correction is not needed.

However, it is worthwhile to note that such correction is possible when better accuracy or a broader range of operating wavelength is needed. In such a case a wavelength-dependent Heydemann correction could be used. In this method the correcting parameters are first calculated as a function of the wavelength and applied later to the Ux and Uy signals. The values of these parameters are selected, based on the instantaneous value of the laser wavelength.

Assuming that the error caused by phase change $\delta \varphi$ less than $87 \mathrm{mrad}$ (i.e. $5^{\circ}$ ) can be corrected by the wavelengthdependent Heydemann correction, the operating wavelength range would reach $\pm 35 \mathrm{~nm}$ around the central value. In practice, it will be smaller due to the dispersion of the LCP bierefringence which must also be taken into account.

In summary, we demonstrated a two-beam polarization interferometer using a $\lambda / 8$ waveplate made from Liquid Crystalline Polymer. The intereferometer was designed to measure small changes in the wavelength of optical radiation emitted by an external cavity laser operating in the range from $628 \mathrm{~nm}$ to $635 \mathrm{~nm}$. Resolution better than $0.002 \mathrm{~nm}$ was attained. The wavelength range of the interferometer can be extended if the wavelengthdependent Heydemann correction is applied to the output signals. The $\lambda / 8$ waveplate can be on the beamsplitter face or on the mirror surface, reducing the cost and complexity of the optomechnical assembly.

The support provided by the Faculty of Electronics, Telecommunication and Informatics at the Gdańsk University of Technology, in the form of DS funding, is gratefully acknowledged.

\section{References}

[1] D. Mawet et al., Opt. Expr. 17, 1902 (2009), http://dx.doi.org/10.1364/OE.17.001902.

[2] G. Myhre et al., Opt. Expr. 20, 27393 (2012), http://dx.doi.org/10.1364/OE.20.027393.

[3] C. Maurer, A. Jesacher, S. Fürhapter, S. Bernet, M. Ritsch-Marte, J. Microscopy 230, 134 (2008), http://dx.doi.org/10.1111/j.1365-2818.2008.01968.x.

[4] P. Juncar, J. Pinard, Rev. Sci. Ins. 53, 939 (1982), http://dx.doi.org/10.1063/1.1137112.

[5] P. Wierzba, M. Gnyba, Eur. Phys. J. ST 154, 235 (2008), http://dx.doi.org/10.1140/epjst/e2008-00552-y.

[6] P.L.M. Heydemann, Appl. Opt. 20, 3382 (1981), http://dx.doi.org/10.1364/AO.20.003382. 